\title{
Understanding the interaction of concanavalin a with mannosyl glycoliposomes: A surface plasmon resonance and fluorescence study
}

\author{
Catalina Sandoval-Altamirano ${ }^{a}$, Susana A. Sanchez ${ }^{b}$, Nancy F. Ferreyrac ${ }^{c}$, \\ German Gunther ${ }^{\mathrm{a}, *}$ \\ a Universidad de Chile, Facultad de Ciencias Químicas y Farmacéuticas, Departamento de Química Orgánica y Fisicoquímica, Casilla 233, Santiago 1, Chile \\ b Universidad de Concepción, Facultad de Ciencias Químicas, Departamento de Polímeros, Concepción, Chile \\ ' INFIQC, Departamento de Fisicoquímica, Facultad de Ciencias Químicas, Universidad Nacional de Córdoba, Córdoba, Argentina
}

\section{A R T I C L E I N F O}

\section{Article history:}

Received 15 March 2017

Received in revised form 7 July 2017

Accepted 8 July 2017

Available online 18 July 2017

\section{Keywords:}

Mannosyl glycoliposomes

Concanavalin A

Surface plasmon resonance

Time-resolved anisotropy

Agglutination

\begin{abstract}
A B S T R A C T
The specificity of carbohydrate-protein interaction is a key factor in many biological processes and it is the foundation of technologies using glycoliposomes in drug delivery. The incorporation of glycolipids in vesicles is expected to increase their specificity toward particular targets such as lectins; however, the degree of exposure of the carbohydrate moiety at the liposome surface is a crucial parameter to be considered in the interaction. Herein we report the synthesis of mannose derivatives with one or two hydrophobic chains of different length, designed with the purpose of modifying the degree of exposure of the mannose when they were incorporated into liposomes.

The interaction of glycovesicles with Con A was studied using: (i) agglutination assays; measured by dynamic laser light scattering (DLS); (ii) time resolved fluorescence methods and (iii) surface plasmon resonance (SPR) kinetic measurements. DLS data showed that an increase in hydrophobic chain length promotes a decrease of liposomes hydrodynamic radius. A longer hydrocarbon chain favors a deeper insertion into the bilayer and mannose moiety results less exposed at the surface to interact with lectin. Fluorescence experiments showed changes in the structure of glycovesicles due to the interaction with the protein. From SPR measurements the kinetic and equilibrium constants associated to the interaction of ConA with the different glycolipid synthetized were determined.

The combination of SPR and fluorescence techniques allowed to study the interaction of Con A with mannosyl glycovesicles at three levels: at the surface, at the interface and deeper into the bilayer.
\end{abstract}

(C) 2017 Elsevier B.V. All rights reserved.

\section{Introduction}

Glycolipids are biomolecules composed of a lipid chain (lipophilic) and a monosaccharide or oligosaccharide as hydrophilic group [1]. The most common glycolipids contain galactose, mannose, fucose, glucose, glucosamine, galactosamine, or sialic acid as sugar in their structure [2]. Most of these compounds are non-toxic, easily degradable and show good solubility in water and organic solvents [3]. Glycolipids are employed in several applications like thermotropic and lyotropic liquid crystals [1,4], surfactants [5], lubricants, pharmaceutical products [6,7], and membrane solubilizing agents, among others.

The amphiphilic nature of glycolipids allows them to spontaneously aggregate to form structures like micelles, bilayers and/or

\footnotetext{
* Corresponding author.

E-mail addresses: ggunther@ciq.uchile.cl, ggunther33@gmail.com (G. Gunther).
}

vesicles. The aggregation capability of glycolipids alkyl polyglycosides, sorbitan esters, and sucrose esters [8-12], have been the fundament for the design of synthetic glycolipids able to selfassemble in innovative structures for their use as drug delivery systems [13-16].

A substantial number of biological processes involving cell-cell interactions such as signaling, recognition and adhesion are modulated by glycans or glycolipids located on the surface of cell as glycoconjugates [17-27], and for this reason glycolipids are considered bioadhesives. Thus, glycolipids are good candidates for drug-targeting, and they have been extensively used to modify the surface of liposomes, niosomes and lipid nanoparticles in order to favor their interactions with specific cells and tissues [24,28-31].

Liposomes are widely used as artificial model for membranes and they have been employed for biochemical and biophysical studies mimicking natural membranes. The main advantage of using liposomes is the feasibility to incorporate different membrane-active agents into the lipid bilayer, creating several 
artificial membrane structures tailored according to the experimental needs. The functionalization of liposomes with glycosylated amphiphiles might increase their specificity toward lectins, a class of nonenzymatic sugar-binding proteins postulated to be involved in several biological processes. Molecular design that guarantees the proper exposure of the saccharide residues on the surface of the lipid nanocarrier is a crucial parameter however it is a difficult issue to be achieved [32].

Mannose is a monosaccharaide present in the membrane of several pathogen microorganisms (bacteria, fungi, protozoa and viruses). This carbohydrate is selectively recognized by the lectin Concanavalin A (Con A), protein widely used to characterize carbohydrate-protein interactions of new designed multivalent systems [29,33-42]. In this work, we studied the interaction using Concanavalin A (Con A) with synthetic liposomes loaded with a series of synthetized mannosyl derivatives. Agglutination, time resolved anisotropy and surface plasmon resonance experiments were used to characterize the protein-carbohydrate interaction. The goal of this work was to evaluate the structural changes of glycovesicles due to the interaction with Con A and to quantitatively characterize the multivalent effect and interaction (kinetic and equilibrium constant) with different glycolipids synthetized. This study shows the relationship between the structure of the mannosyl derivative and the interaction of liposomes (containing the derivatives) with Con A. Besides, this report also shows the changes produced in the bilayer as a consequence of inserting mannosyl derivatives with different structure.

\section{Materials and methods}

\subsection{Reagents}

Octanol, dodecanol, hexadecanol, 1-bromooctane, 1bromododecane, 1-bromohexadecane, $\mathrm{BF}_{3} \mathrm{OEt}_{2}, \mathrm{SnCl}_{4}(1 \mathrm{M}$ in $\mathrm{CH}_{2} \mathrm{Cl}_{2}$ ), 1.6-diphenyl-1,3,5-hexatriene (DPH), glycerol, mannose, palmitoyl-oleoyl-phosphatidylcholine (POPC), dipalmitoylphosphatidylcholine (DPPC), Concanavalin A (Con A), Bovine serum albumin (BSA), mercapto-11-undecanol (MUO) from Sigma-Aldrich and Laurdan from Molecular Probes, were employed without further purification. Dioctadecyl-trimethylammonium chloride (DODAC) technical grade from Herga Industries was extracted and recrystallized six times from acetone, as described previously [43]. All solvents (Merck) were dried over molecular sieves of $3 \AA \AA$ or $4 \AA$, for at least $48 \mathrm{~h}$. All measurements were performed at controlled temperature with a HAAKE $\mathrm{F} 3$ thermostated bath and an OMEGAETTE thermocouple.

\subsection{Unilamellar vesicle preparation}

Unilamellar vesicles were prepared by sonication and extrusion methods. The lipids or lipid mixture were dissolved in a volatile solvent (methanol or chloroform) in the desired ratio to obtain homogeneous mixtures. The solvent was removed under nitrogen flow and the film was suspended and re-hydrated in saline HEPES buffer ( $\mathrm{pH}$ 7.4, $10 \mathrm{mM}$ HEPES, $10 \mathrm{mM} \mathrm{NaCl}, 0.1 \mathrm{mM}$ de $\mathrm{CaCl}_{2}$ and $\mathrm{MnCl}_{2}$ ), and ultra-sonicated with a Liposomicator (Avanti lipids). When necessary, previously prepared unilamellar vesicles were extruded at $60^{\circ} \mathrm{C}$ through a $50 \mathrm{~nm}$ polycarbonate membrane in a $10 \mathrm{~mL}$ thermostated extruder (Thermobarrel extruder, from Northern Lipids) to obtain vesicles with specific size and narrow size distributions [44].

\subsection{Agglutination assays}

Liposome aggregation induced by Con A was studied by adding the lectin to the solutions of liposomes, keeping a lipid/Con A molar ratio equal to 100 , and stirring for $48 \mathrm{~h}$ at $25^{\circ} \mathrm{C}$. Solutions were prepared with saline HEPES buffer ( $\mathrm{pH} 7.4,10 \mathrm{mM}$ HEPES, $10 \mathrm{mM}$ $\mathrm{NaCl}, 0.1 \mathrm{mM}$ de $\mathrm{CaCl}_{2}$ and $\mathrm{MnCl}_{2}$ ). The aggregation process was monitored using Dynamic Light Scattering (DLS, Zetasizer Nano ZS, Malvern), equipped with a green laser of $532 \mathrm{~nm}$ or turbidity measurements (UV-vis, 8453 spectrophotometer, Agilent).

\subsection{Time resolved fluorescence and anisotropy measurements}

The selected probes, Laurdan and DPH, were incorporated into liposomes from an ethanolic solution, heated and maintained above lipid transition temperature $\left(60^{\circ} \mathrm{C}\right)$ during one hour. Temperature was reduced to $25^{\circ} \mathrm{C}$ and Con $\mathrm{A}$ was added keeping the lipid/Con $A$ ratio equal to 100 and the solution stirred for $48 \mathrm{~h}$. These experiments were carried out using the saline HEPES buffer previously described.

Time resolved fluorescence measurements were performed with a Fluotime 200 (PicoQuant), using time correlated single photon counting method (TCSPC) with a multichannel Timeharp 250 card (PicoQuant). As excitation source, an $80 \mathrm{MHZ}$ laser of $375 \mathrm{~nm}$ was employed. The emissions of Laurdan and DPH were monitored at 440 and $450 \mathrm{~nm}$ respectively.

The decays corresponding to time resolved anisotropy (TRA) were fitted using the wobbling in a cone model proposed by Kinosita, K. et al. [45]. that considers a residual anisotropy, the probe is located in an environment where rotational diffusion is restricted and complete depolarization is not attainable (Eq. (1)) [45]. The model describes the rotation of the probe in the bilayer as a wobble in a cone which has a characteristic angle $(\theta$, related to the molecular order of the bilayer) and a rotation time ( $\tau$, associated to dynamic movements on the membrane: lateral diffusion, rotational, isomerization, among others) depending on the characteristics of the lipid system (Eq. (2)). The multiplicity of the factors involved makes difficult to explain the variations in the second parameter [46]. (An example of the fitting is showed in Fig. S2).

$$
\begin{aligned}
& r(t)=\left(r_{0}-r_{\infty}\right) e^{-\frac{t}{\tau_{r}}}+r_{\infty} \\
& \frac{r_{\infty}}{r_{0}}=\frac{3 \cos ^{2} \theta-1^{2}}{2}
\end{aligned}
$$

\subsection{Surface plasmon resonance (SPR) experiments}

\subsubsection{Surface preparation}

All experiments were carried out on a dual channel SPR 7500DC (Reichert) using gold chips functionalized with MUO self-assembled monolayer (SAM). This surface modification was performed ex situ by immersion of the chip during $48 \mathrm{~h}$ in a $1 \mathrm{M}$ ethanolic solution of MUO. At the end of the adsorption step the substrate was exhaustively cleaned with ethanol and dried under nitrogen flow. Before start the measure, the working channel was modified with a $3 \mathrm{mM}$ liposome solution (total lipid) at a $5 \mu \mathrm{L} / \mathrm{min}$ flow during $40 \mathrm{~min}$. To eliminate weakly adsorbed liposomes, buffer solution was injected at a flow rate of $250 \mu \mathrm{L} / \mathrm{min}$ during $10 \mathrm{~min}$. A $100 \mathrm{mM}$ phosphoric acid solution was injected as the final cleaning step. Binding experiments: Con A solutions of the desired concentration prepared in HEPES saline buffer with $0.5 \mathrm{mg} / \mathrm{ml}$ de BSA were injected to the cell at a flow of $50 \mu \mathrm{L} / \mathrm{min}$. BSA was added to the buffer solution to prevent possible unspecific interactions between Con A and the monolayer of MUO. Con A solution flow rate was previously selected performing a test of mass transport. Between binding cycles, the lipid coated surface was regenerated with $100 \mathrm{mM}$ phosphoric acid solution in running buffer. Experimental data were corrected for instrumental and bulk artifacts by double referencing, a control sensor chip surface (channel two) and buffer injections using Integrated SPRAutolink 


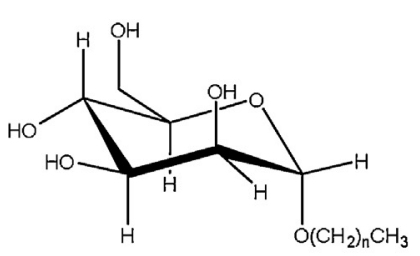

$\operatorname{MOM}(\mathrm{n}=8), \operatorname{MLM}(\mathrm{n}=12), \operatorname{MPM}(\mathrm{n}=16)$

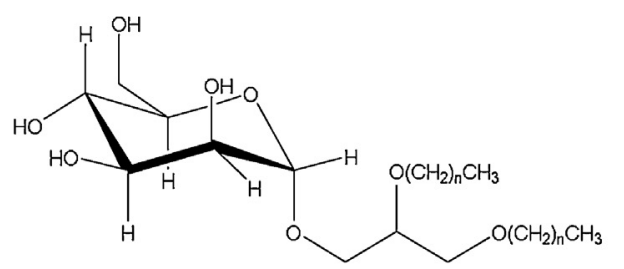

$\operatorname{DOM}(\mathrm{n}=8), \operatorname{DLM}(\mathrm{n}=12), \operatorname{DPM}(\mathrm{n}=16)$

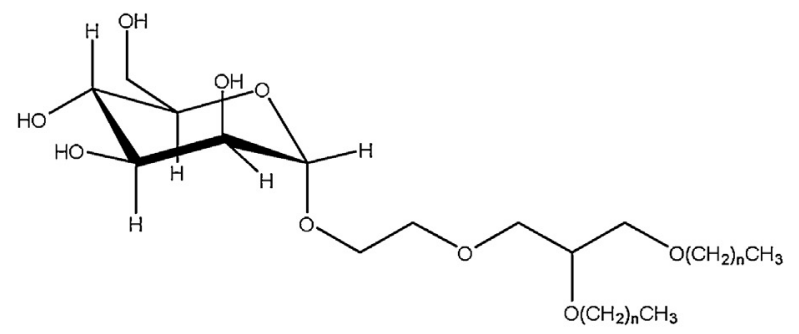

$\operatorname{DOEM}(\mathrm{n}=8), \operatorname{DLEM}(\mathrm{n}=12), \operatorname{DPEM}(\mathrm{n}=16)$

Fig. 1. Chemical structure of the alkyl mannosyl derivatives synthetized.

(Reichert, USA). Data analysis was performed with OriginLab ${ }^{\circledR} 8.0$ software.

\subsubsection{Test for mass transport effects}

Binding tests at different, flow rates $25,50,75$ and $100 \mu \mathrm{L} / \mathrm{min}$, were performed with a Con A $0.026 \mathrm{mM}$ solution to confirm the absence of mass transport effects influencing the shape of the sensorgrams. A visual inspection of the overlapped sensorgrams demonstrated that the transport of the lectin to the sensor surface does not influence the kinetics of the binding between the immobilized glycolipid and Con A. From this, test a working flow of $50 \mu \mathrm{L} / \mathrm{min}$ of Con A solution was selected.

\section{Results and discussion}

In order to study the interaction of Con A and glycolipids we synthesized several mannosyl derivatives with one (MXM) or two (DXM and DXEM) chains of different length (Fig. 1). The experimental design consisted on forming vesicles having a lipid as a main matrix and different percentage of the mannosyl glycolipid. Three different commercial lipids were used as possible candidates to form the matrix, one cationic (DODAC) and two zwitterionic phospholipids (DPPC and POPC).

\subsection{Selection of the appropriate lipid to form the matriz of the liposome}

The lipid to form the matrix should show minimal interaction with Con A. To test the existence of possible interactions between the lipid used to form the matrix and Con A, agglutination assays and time resolved anisotropy (TRA) measurements were performed on vesicles without glycolipids in the presence and absence of Con A. The results of the agglutination assays (Fig. S1, supplementary information) showed no significant changes in the hydrodynamic diameter of neither POPC nor DPPC vesicles, but a notorious increase of the hydrodynamic diameter for DODAC vesicles. This behavior may have its origin in the positive surface charge of the ammonium head of DODAC. Favorable interactions may be established due to opposite surface charge (zeta potential DODAC $80 \mathrm{mV}$ and Con $\mathrm{A}-10 \mathrm{mV})$. The zeta potential of DPPC $(-13.1 \mathrm{mV})$ and POPC liposomes $(-6.5 \mathrm{mV})$ prevents the unspecific interactions and the agglutination process [47].
Time anisotropy for DPH and Laurdan were used to evaluate the changes in the order parameter of the bilayer due to the interaction with Con A. DPH reports the order in the hydrocarbon chain region and Laurdan on the water accessibility at the interface [48]. In the presence of Con A, a decrease in the area occupied by the phospholipids is observed (lower $\theta$ angles for DPH and Laurdan, Fig. S3). While an increase in viscosity at the hydrocarbon chain region and a decrease in viscosity at the interface is observed (growth in $\tau_{\mathrm{r}}$ of DPH and decrease in $\tau_{\mathrm{r}}$ for Laurdan Fig. S3). The lowest change in $\theta$ and $\tau_{\mathrm{r}}$ for DPH and Laurdan due to presence of Con A was observed for DPPC liposomes indicating thus the lowest perturbation of the liposome structure.

The mild changes observed in the hydrodynamic diameter and the membrane properties for DPPC in the presence of Con A indicated a negligible unspecific interaction of this lipid with Con A. Therefore, this phospholipid was selected to be used as lipid matrix to incorporate the synthesized mannosyl derivatives and to study their interaction with Con $\mathrm{A}$.

\subsection{Selection of the percentage of mannosyl derivatives to be incorporated to DPPC liposomes}

The presence of Con A, has been reported to favor the fusion of vesicles containing mannose or glucose glycolipids to produce aggregation and subsequent 'coalescence' of the liposomes $[26,28,34,49]$. This phenomenon can be detected by the increase in optical density of the suspensions as well as from the variation of the particle size determined by DLS. Fig. 2 shows the changes in optical density produced by the addition of Con A to DPPC liposomes prepared with increasing percentage $(0,1,5$ and $10 \%)$ of the glycolipid DPEM. No variations in optical density were observed during the evaluated time for the control experiment corresponding to Con A solution. First, we measured the changes in optical density produce by the interaction of the lectin with the pure DPPC liposomes. An increase in optical density was observed; reaching a plateau at $100 \mathrm{~min}$, Addition of DPEM to the DPPC liposomes produced the largest changes at $5 \% \mathrm{~mol}$ (Fig. 2). Further increment of DPEM (10\%) resulted in a decrease of the scattering. perhaps due to the formation of lipid domains with the concomitant reduction in the number of mannose units available to interact with Con A. It may also be a decrease of zeta potential of the vesicles with the consequent increment of the electrostatic repulsion with the 


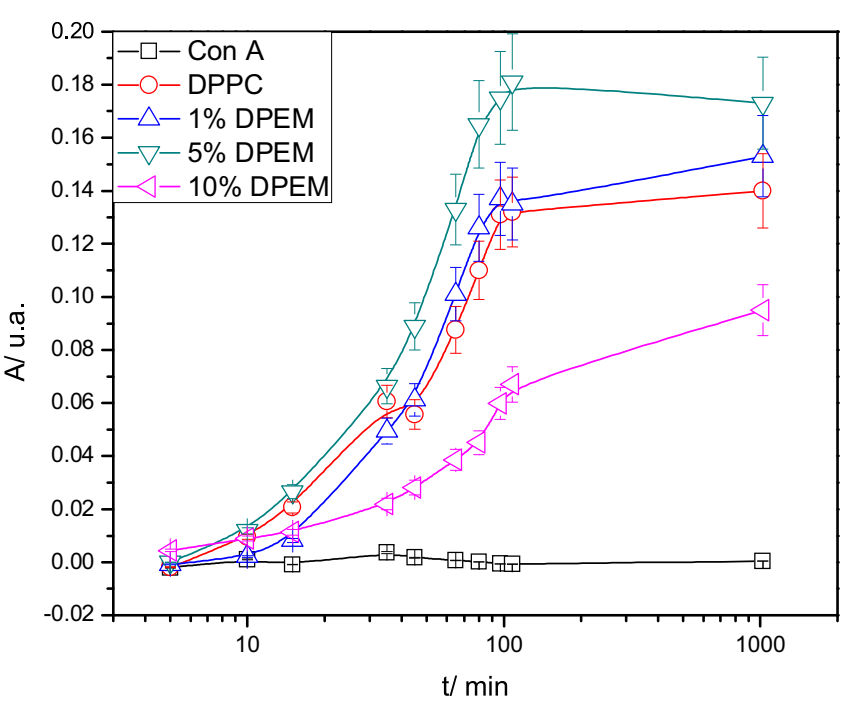

Fig. 2. Optical density change (followed at $400 \mathrm{~nm}$ ) promoted by the presence of Con A incubated with DPPC liposomes containing increasing amounts of DPEM. $($ Mean $\pm S D ; n=3)$.

protein [50]. Considering these results DPPC liposomes containing $5 \%$ mol of glycolipids were used in the rest of the experiments.

\subsection{Evaluation of the interactions glycolipids-ConA}

The interaction of the glycovesicles (DPPC with $5 \%$ of mannosyl derivatives) with Con A was studied using agglutination assays, TRA and SPR.

\subsubsection{Agglutination assays}

The multivalent interaction of these systems favors the formation of three-dimensional networks and hence, a significant increase in size would be expected upon aggregation. Fig. 3 shows the change of hydrodynamic diameter, $\Delta \mathrm{d}$, for the different glycovesicles incubated with Con A. The response depends on the nature of the glycolipid, and is related to the accessibility of the carbohydrate on the surface of the liposome to reach the protein active site.

The effect of the length of hydrocarbon chain was studied by comparing the synthetized glycolipids by family. In the case of

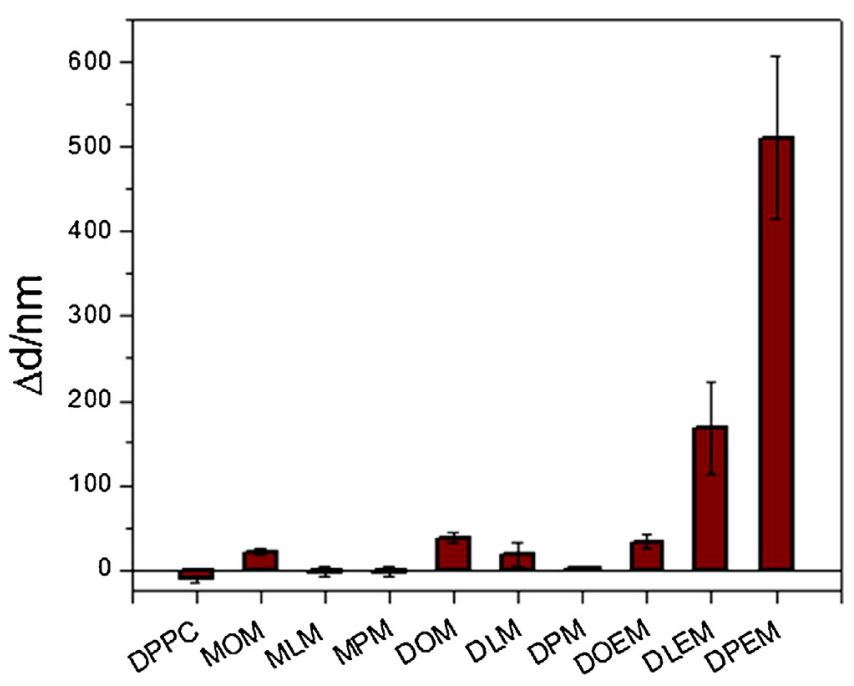

Fig. 3. Increase of the particle size observed upon addition of Con A to DPPC liposomes loaded with $5 \%$ of mannose derivatives. (Mean $\pm S D ; n=5$ ).
MXM (derivatives with one alkyl chain: MOM, MLM and MPM), the increase in chain size promoted a decrease of $\Delta \mathrm{d}$. Derivatives with long chains showed a decrease of the hydrodynamic radius, however this reduction was not as much as the one observed in absence of mannosides (Fig. 3). Similar trend was observed with the DXM derivatives with two alkyl chains (DOM, DLM and DPM), there is an increase in size, but its magnitude is inversely proportional to alkyl chain length. These results can be understood considering the anchoring of the glycolipids into the DPPC bilayer depends on the length of the carbon chain, a longer carbon chain would favor deeper insertion of the glycolipid into the bilayer, leaving the mannose moiety less exposed to interact with the lectin. Therefore, the anchoring of the glycolipid would determine the degree of exposition of the mannose moiety and thus the capability of interaction with Con A.

A different situation occurred when an hydrophilic extensor was added to the molecule. For DXEM an increase in the hydrocarbon chain length produces an increment of the hydrodynamic radius of the particles formed after the interaction with Con A. The most notorious changes were observed with DLEM and DPEM (Fig. 3). The presence of the spacer promoted the exposure of mannose, the interaction with Con A and the formation of larger particles. This effect (presence of an ethylenglycol spacer) was studied by Engel et. al [51]. and they proposed an hydrocarbon chain with 16 methylene units being the optimal spacer. They reported that spacer with length over six units would decrease the interaction with Con A due to modifications in the arrangements and folding modes of the spacer chain.

\subsubsection{Time-resolved fluorescence anisotropy (TRA) studies}

Mannose moiety can have different degrees of exposure in the liposome, depending on the type of glycolipids being inserted. Therefore, it is reasonable to expect a dissimilar membrane structuration being promoted by the insertion of different type of glycolipid in the liposome. If the hydrophilic head of the glycolipid sticks out from the surface of the vesicle, the interaction with Con A would not be expected to produce significant changes in the membrane parameters. On the other hand, if the polar head of the glycolipids sinks deeper into the interface, membrane structure would be affected.

Fig. 4 shows the analysis for the TRA data for Laurdan and DPH of all derivatives studied. As a general observation, the wobbling angle $(\theta)$ increased for DPH and a decreased for Laurdan, when Con A was added to the liposome suspension. These results indicate that Con A may promote an increase in lipid chain cross-section and a decrease in the molecular area at the interface in the membrane.

For DPPC-DOEM glycoliposomes, no changes in the wobbling angle for any of the two probes were detected. This result can be explained if mannose moiety is fully exposed, pocked out, on the liposome interface, and there is no direct contact between the protein and liposome surface. In the case of DXEM glycolipids with 12 and 16 methylene units, the increase in hydrophobicity of hydrocarbon chains causes the glycolipids to be incorporated deeper into the membrane, (but still exposed) and the interaction with Con $\mathrm{A}$ occurs closer to the surface, resulting in an increase of $\theta$ for DPH and a decrease of $\theta$ for Laurdan. We hypothesize that during the interaction with glycoliposomes containing mannosyl derivatives with 12 and 16 methylene units, there is a region of contact between the protein and the interface of liposome, which promotes structural modifications on membrane.

\subsubsection{Surface plasmon resonance}

SPR experiments were performed to evaluate interaction of pure DPPC liposomes and DPPC liposomes with 5\% mol of DLEM or DPEM. We selected these glycolipids, according with the previous results due to the better exposition of mannose for the interaction with 

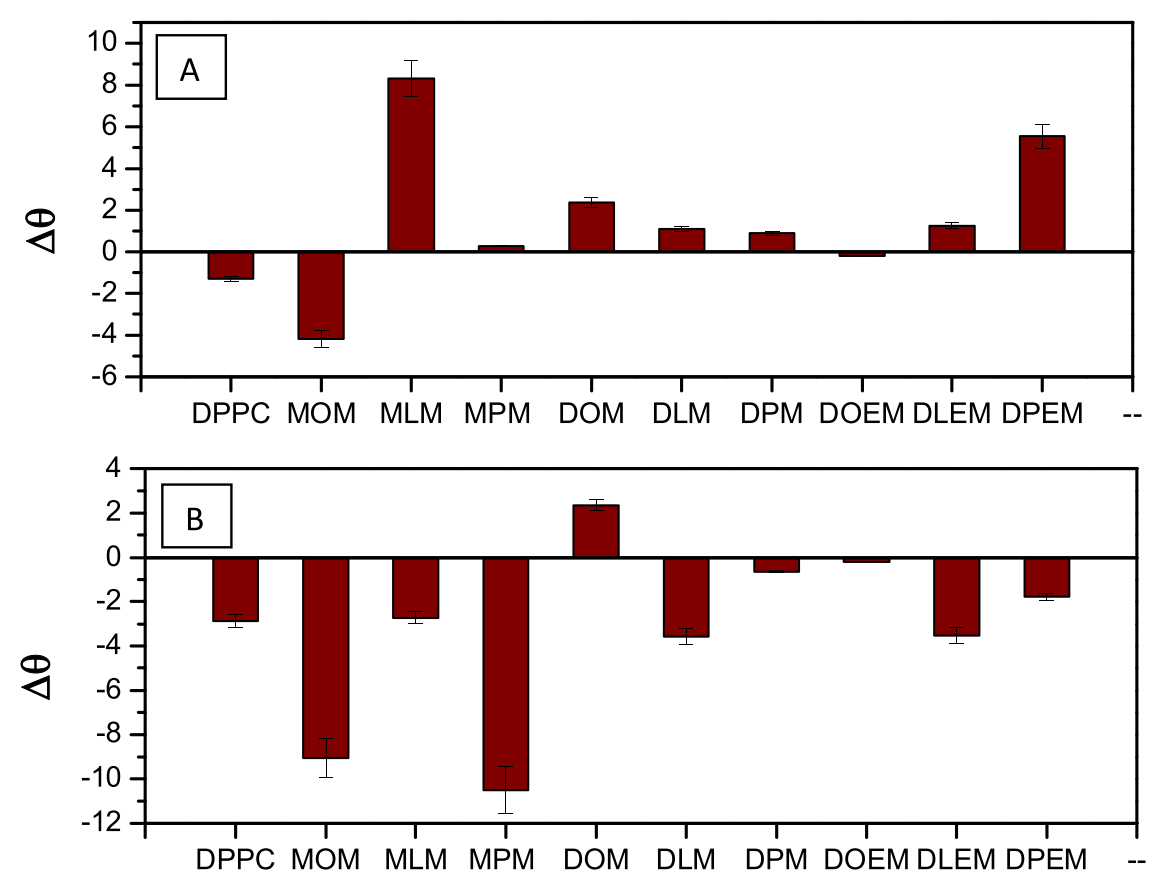

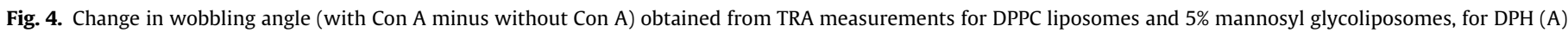
and for Laurdan (B). (Mean $\pm S D ; n=3)$.

lectin. We immobilized the liposomes on MUO SAMs (Fig. S4 of supplementary information) and we evaluate the interaction with increasing concentrations of Con A. After a test for possible mass transport limitations, Fig. S5, we evaluated the interaction of Con A with pure DPPC liposomes. Fig. S6 presents the sensorgrams for the interaction of Con A with pure DPPC liposomes. We determined the kinetic constants for this interaction from a global fit of these curves with an $1: 1$ model $\left(\mathrm{k}_{\mathrm{a}}=(0.70 \pm 0.02) \times 10^{4} \mathrm{M}^{-1} \mathrm{~s}^{-1}\right.$ and $\mathrm{k}_{\text {off }}$ $\left.(3.0 \pm 0.1) \times 10^{-3} \mathrm{~s}^{-1}\right)$.

The response observed due to interaction with Con $A$ in pure DPPC, when compared with the sensorgrams obtained with the liposomes containing 5\% mole of DLEM (Fig. 5A) and 5\% mole of DPEM (Fig. 5B), shows notorious differences. At concentrations of Con A comparable with those employed for pure DPPC, the SPR signals of the interaction of Con A - glycoliposomes immobilized are higher, and no saturation is reached in the interval of Con A concentration analyzed (up to $7.8 \times 10^{-6} \mathrm{M}$ ). The fitting analysis indicates a complex binding profile with a fast-initial interaction is observed.

The binding mechanism for the interaction between glycoliposomes and Con A depends on the concentration of mannose moieties at surface [52]. A multivalent effect is expected at low concentration, a combination between monovalent and bivalent interaction will be observed as an intermediate situation and at higher concentration monovalent effect will predominate due to steric effects [53]. Considering the size of the glycoliposomes and the estimated surface density of DPEM and DLEM molecules in our experiments, the maximum theoretical average distance between glycolipids will be $3.5 \mathrm{~nm}$. Being the distance between carbohydrate recognition sites of Con A $6.5 \mathrm{~nm}$, thus we considered the occurrence of multivalent interaction of the glycoliposomes with Con A. Accordingly, a cluster interaction of Con A with the mannose derivatives and an enhancement of Con A binding to mannosyl ligands should be expected [54].

The association phase in the sensorgrams of Fig. $5 \mathrm{~A}$ and $\mathrm{B}$ shows a pronounced initial slope, indicating an initial fast-rate binding. Under this considerations we followed E. M. Muñoz et al., procedure to perform the kinetic analysis [52]. We analyzed the late dissociation phase $\left(t_{0}>170 \mathrm{~s}\right)$, through a global fit with the classical equation for pseudo-first-order kinetics. From this, we determined the apparent dissociation rate constant $\mathrm{k}_{\text {off }}$ that accounts for the release of Con A from the glycoliposomes surface stabilized by a multivalent effect. The evaluation of the early association phase was done by non-linear analysis using the pseudo-first-order kinetic equations but deviations from this behavior were observed due to the binding heterogeneity of the systems. The plots of $\mathrm{k}_{\mathrm{obs}}$ vs [Con A] presented two well-defined slopes (Fig. S6 in supplementary information). The largest slope (faster association) was observed at low Con A concentration indicating the presence of higher order complexes in the low Con A range. From global fitting of the pseudo first-order rate equation (Eq. (3)) at the early association phase $(t<14 \mathrm{~s}$ for high Con A concentration and $\mathrm{t}<25 \mathrm{~s}$ for low Con A concentration), we estimated $\mathrm{ka}_{1}$ for low concentration of Con $A$ and $\mathrm{k}_{\mathrm{a} 2}$ for high concentration of Con $\mathrm{A}$, which are related with multi and monovalent processes respectively.

$R_{t}=R_{e q}\left(1-\exp ^{-\left(k_{a}[\operatorname{Con} A]+k_{o f f}\right) t}\right)(3)$

As an intrinsic dissociation rate constant cannot be defined, we used the previously obtained $\mathrm{k}_{\text {off }}$ values. The association constant $\left(\mathrm{K}_{\mathrm{a}}\right)$ was estimated from the early association and late dissociation kinetic data.

As shown Table 1, the magnitude of the apparent association rate constants (determined at the same percentage of mannosyl derivatives, for the comparative analysis) depends on the glycolipid nature. This lectin has a higher affinity for glycoliposomes than for pure DPPC liposomes, difference associated to the higher values of the rate association constant for the liposomes containing the mannosyl derivatives. Consequently, the values determined for the kinetic rate constants ka and $\mathrm{k}_{\text {off }}$ are apparent values for the interaction of cluster domains of DLEM or DPEM in the environment provided by DPPC. The greater apparent association constant $\mathrm{k}_{\mathrm{a} 1}$ for DPEM glycoliposomes than for DLEM glycoliposomes is consistent with the results obtained in agglomeration studies. In addition, Ka value for DPEM glycoliposomes is higher than for DLEM glycoliposomes, consequently sensorgrams obtained with equivalent concentration of Con A produce higher SPR signals. The estimated 

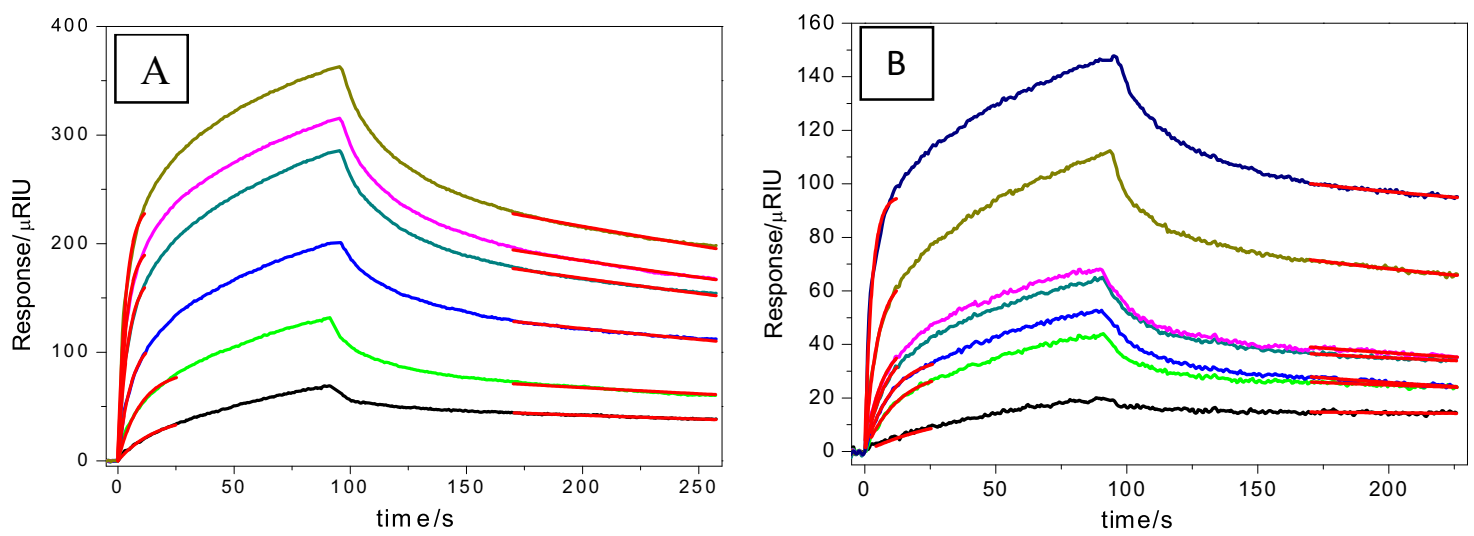

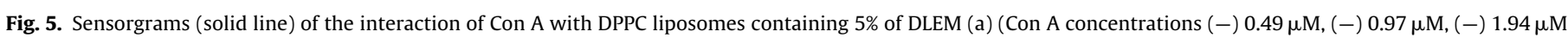

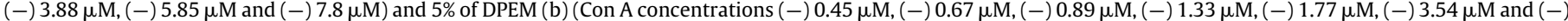
$7.11 \mu \mathrm{M})$.

Table 1

Association and dissociation rate constants for the interaction between immobilized DPPC liposomes and Con A. (Mean \pm SD; $\mathrm{n}=3$ ).

\begin{tabular}{|c|c|c|c|c|}
\hline & $\mathrm{k}_{\mathrm{a} 1} / 10^{4} \mathrm{M}^{-1} \mathrm{~s}^{-1}$ & $\mathrm{k}_{\mathrm{a} 2} / 10^{4} \mathrm{M}^{-1} \mathrm{~s}^{-1}$ & $\mathrm{k}_{\mathrm{off}} / 10^{-3} \mathrm{~s}^{-1}$ & $\mathrm{~K}_{\mathrm{a}} / 10^{7} \mathrm{M}^{-1}$ Low [Con $\left.\mathrm{A}\right]$ \\
\hline 5\% DLEM & $8.5 \pm 0.7$ & $2.2 \pm 1.0$ & $1.7 \pm 0.3$ & $5 \pm 1$ \\
\hline 5\% DPEM & $13 \pm 3.0$ & $4.3 \pm 0.8$ & $1.3 \pm 0.3$ & $10.4 \pm 0.3$ \\
\hline
\end{tabular}

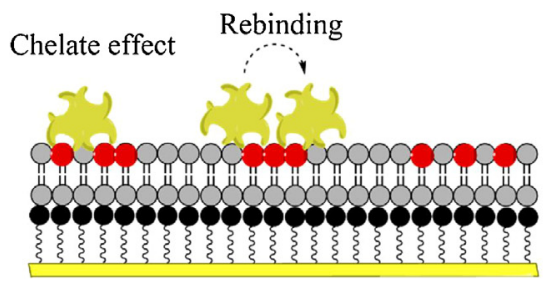

$\operatorname{Low}[\operatorname{Con} A]$

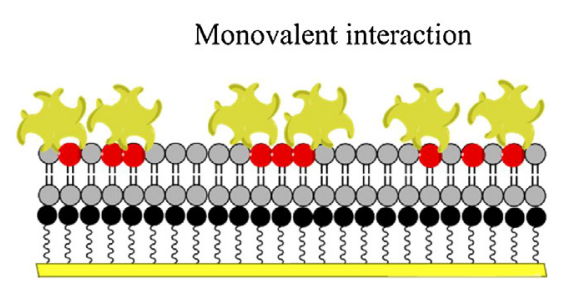

High [Con A]

Fig. 6. Scheme interaction as a function of Con A concentration.

apparent affinity constant values for the glycoliposomes containing DLEM and DPEM are higher than those reported for simple sugar recognition (i.e.monovalent $\alpha$-mannopyranose) which usually are in a very low affinity range (about $10^{3} \mathrm{M}^{-1}$ )[40] for the interaction with Con A. Our results are consistent with those reported for multivalent systems such as polymers and dendrimers having mannose side chains (Ka around $1.0 \times 10^{7} \mathrm{M}^{1}$ )[53,55-57].

An initial phase characterized by two kinetic constants could be explained because at low Con A concentrations multivalent effect predominates in the interaction, but increasing Con A concentration, monovalent interactions prevail due to steric hindrance, as depicted in Fig. 6. The multivalent effect can be attributed to several processes; intermolecular aggregation, intramolecular chelation, steric stabilization of the surface or statistical rebinding [58]. These effects depends on the surface concentration and organization of the monosaccharide as well as concentration of the multivalent ligand (ConA). The statistical rebinding can be explaining due to the proximity of mannosyl groups in the surface; the association of Con A with a mannosyl moiety can occur immediately after dissociation of the same Con A with other mannosyl in the proximity. If the statistical rebinding is operative in these systems, our results indicate that DPEM molecules are closer than DLEM molecules in the bilayer (formation of domains where DPEM is enriched).
This study of the interaction with Con A not only allows us to elucidate the multivalent behavior of glycoliposomes, but also provides information on the organization of glycolipids in bilayers (i.e domain formation).

\section{Conclusions}

The main topic of this article is to establish how the degree of exposure of mannose moiety at the surface of the liposome influences its recognition of glycoliposomes by Concanavalin $\mathrm{A}$

Our results indicate that for longer methylene chains in mono and dialkyl derivatives the exposure of the mannose moiety at the surface of the liposome decreases, while the presence of one oxyethylene unit as extender reverts this behavior. SPR determinations indicate the existence of a complex mechanism for the interaction of Con A with glycoliposomes; depending on Con A concentrations, a fast-initial interaction was attributed to multivalent process and a slow interaction was related with monovalent processes. Around 1,5 time increase on the association constant was observed at the lower concentration of Con A for glycoliposomes with 5\% DPEM indicating the prevalence of a multivalent effect under this condition and therefore a greater affinity constant of this derivative for Con $\mathrm{A}$. 


\section{Acknowledgments}

The authors thank Fondecyt-Chile1120196(G.G.) 1140454 (S.S), CONICYT-FONDEQUIP (EQM140112), N. Ferreyra thanks to SECyTUNC and Agencia Nacional de Promoción Científica y Tecnológica (ANPCYT) and for the financial support. C. Sandoval-Altamirano thanks to Conicyt Beca Doctorado Nacional 21120554

\section{Appendix A. Supplementary data}

Supplementary data associated with this article can be found, in the online version, at http://dx.doi.org/10.1016/j.colsurfb.2017.07. 026.

\section{References}

[1] V. Faivre, V. Rosilio, Interest of glycolipids in drug delivery: from physicochemical properties to drug targeting, Expert Opin. Drug Deliv. 7 (2010) 1031-1048.

[2] R.A. Dwek, Glycobiology Toward understanding the function of sugars, Chem. Rev. 96 (1996) 683-720.

[3] V. Vill, R. Hashim, Carbohydrate liquid crystals: structure-property relationship of thermotropic and lyotropic glycolipids, Curr. Opin. Colloid Interfaces 7 (2002) 395-409.

[4] S. Gerber, M. Wulf, G. Milkereit, V. Vill, J. Howe, M. Roessle, P. Garidel, T. Gutsmann, K. Brandenburg, Phase diagrams of monoacylated amide-linked disaccharide glycolipids, Chem. Phys. Lipids 158 (2009) 118-130.

[5] F.A. Sani, T. Heidelberg, R. Hashim, Farhanullah, Alkyl triazole glycosides (ATGs)-A new class of bio-related surfactants, Colloids Surf. B 97 (2012) 196-200.

[6] T. Morita, T. Fukuoka, T. Imura, D. Kitamoto, Production of mannosylerythritol lipids and their application in cosmetics, Appl. Microbiol. Biotechnol. 97 (2013) 4691-4700.

[7] T. Morita, T. Fukuoka, T. Imura, D. Kitamoto, Mannosylerythritol lipids: production and applications, J. Oleo Sci. 64 (2015) 133-141.

[8] J.M. Hierrezuelo, J.A. Molina-Bolivar, C. Carnero-Ruiz, On the urea action mechanism: a comparative study on the self-assembly of two sugar-based surfactants, J. Phys. Chem. B 113 (2009) 7178-7187.

[9] M.J. Donnelly, J.D. Bu'Lock, Sucrose esters as model compounds for synthetic glycolipid suffactants, J. Am. Oil Chem. Soc. 65 (1988) 284-287.

[10] N. Becerra, C. Toro, A.L. Zanocco, E. Lemp, G. Günther, Characterization of micelles formed by sucrose 6-O-monoesters, Colloids Surf. A 327 (2008) 134-139.

[11] K. Hill, O. Rhode, Sugar-based surfactants for consumer products and technical applications, Fett-Lipid 101 (1999) 25-33.

[12] J.P. Gunjikar, A.M. Ware, S.A. Momin, Evaluation of different alkyl polyglycoside surfactants and their combination with alpha olefin sulphonate for detergency, J. Disper. Sci. Technol. 27 (2006) 265-269.

[13] G. Godeau, P. Barthelemy, Glycosyl-nucleoside lipids as low-molecular-weight gelators, Langmuir 25 (2009) 8447-8450.

[14] G. Godeau, C. Brun, H. Arnion, C. Staedel, P. Barthelemy, Glycosyl-nucleoside fluorinated amphiphiles as components of nanostructured hydrogels, Tetrahedron Lett. 51 (2010) 1012-1015.

[15] T. Abraham, M. Hato, M. Hirai, Polymer-dispersed bicontinuous cubic glycolipid nanoparticles, Biotechnol. Progr. 21 (2005) 255-262.

[16] N. Ahmad, R. Ramsch, J. Esquena, C. Solans, H.A. Tajuddin, R. Hashim, Physicochemical characterization of natural-like branched-chain glycosides toward formation of hexosomes and vesicles, Langmuir 28 (2012) 2395-2403.

[17] R. Chammas, S.S. Veiga, S. Line, P. Potocnjak, R.R. Brentani, Asn-linked oligosaccharide-dependent interaction between laminin and Gp120/140 - an alpha-6/Beta-1 integrin, J. Biol. Chem. 266 (1991) 3349-3355.

[18] G. Opdenakker, P.M. Rudd, C.P. Ponting, R.A. Dwek, Concepts and principles of glycobiology, FASEB J. 7 (1993) 1330-1337.

[19] M.Z. Zheng, H. Fang, T. Tsuruoka, T. Tsuji, T. Sasaki, S. Hakomori, Regulatory role of Gm3 ganglioside in alpha-5-beta-1 integrin receptor for fibronectin-mediated adhesion of Fua169Cells, J. Biol. Chem. 268 (1993) 2217-2222

[20] R. Mody, S. Joshi, W. Chaney, Use of lectins as diagnostic and therapeutic tools for cancer, J. Pharmacol. Toxicol. 33 (1995) 1-10.

[21] M.D. Disney, P.H. Seeberger, The use of carbohydrate microarrays to study carbohydrate-cell interactions and to detect pathogens, Chem. Biol. 11 (2004) 1701-1707.

[22] L. Nimrichter, A. Gargir, M. Gortler, R.T. Altstock, A. Shtevi, O. Weisshaus, E. Fire, N. Dotan, R.L. Schnaar, Intact cell adhesion to glycan microarrays, Glycobiology 14 (2004) 197-203.

[23] B. Blomme, C. Van Steenkiste, N. Callewaert, H. Van Vlierberghe, Alteration of protein glycosylation in liver diseases, J. Hepatol. 50 (2009) 592-603.

[24] B. Mukhopadhyay, M.B. Martins, R. Karamanska, D.A. Russell, R.A. Field, Bacterial detection using carbohydrate-functionalised CdS quantum dots: a model study exploiting E. coli recognition of mannosides, Tetrahedron Lett. 50 (2009) 886-889.
[25] N.E. Zachara, G.W. Hart, Cell signaling, the essential role of O-GlcNAc! BBA-Mol. Cell Biol. Lip. 1761 (2006) 599-617.

[26] A. Mauceri, A. Fracassi, M. D’Abramo, S. Borocci, L. Giansanti, A. Piozzi, L. Galantini, A. Martino, V. D'Aiuto, G. Mancini, Role of the hydrophilic spacer of glucosylated amphiphiles included in liposome formulations in the recognition of Concanavalin A, Colloids Surf. B 136 (2015) 232-239.

[27] A. Imberty, A. Varrot, Microbial recognition of human cell surface glycoconjugates, Curr. Opin. Struct. Biol. 18 (2008) 567-576.

[28] B.S. Kim, D.J. Hong, J. Bae, M. Lee, Controlled self-assembly of carbohydrate conjugate rod-coil amphiphiles for supramolecular multivalent ligands, J. Am. Chem. Soc. 127 (2005) 16333-16337.

[29] C.C. Huang, C.T. Chen, Y.C. Shiang, Z.H. Lin, H.T. Chang, Synthesis of fluorescent carbohydrate-protected Au nanodots for detection of concanavalin a and escherichia coli, Anal. Chem. 81 (2009) 875-882.

[30] A.K. Michel, P. Nangia-Makker, A. Raz, M.J. Cloninger, Lactose-functionalized dendrimers arbitrate the interaction of galectin-3/MUC1 mediated cancer cellular aggregation, Chembiochem 15 (2014) 2106-2112.

[31] C.K. Goodman, M.L. Wolfenden, P. Nangia-Makker, A.K. Michel, A. Raz, M.J. Cloninger, Multivalent scaffolds induce galectin-3 aggregation into nanoparticles, Beilstein J. Org. Chem. 10 (2014) 1570-1577.

[32] A. Makky, J.P. Michel, P. Maillard, V. Rosilio, Biomimetic liposomes and planar supported bilayers for the assessment of glycodendrimeric porphyrins interaction with an immobilized lectin, BBA Biomembr. 1808 (2011) 656-666.

[33] G.N. Reeke, J.W. Becker, B.A. Cunningham, J.L. Wang, I. Yahara, G.M. Edelman, Structure and function of concanavalin a, in: T.K. Chowdhury, A.K. Weiss (Eds.), Concanavalin A, Springer, US, Boston, MA, 1975, pp. 13-33.

[34] R. Sundler, Agglutination of glycolipid-phospholipid vesicles by concanavalin A: Evidence for steric modulation of lectin binding by phospholipid head groups, FEBS Lett. 141 (1982) 11-13.

[35] T. Sanji, K. Shiraishi, M. Nakamura, M. Tanaka, Fluorescence turn-on sensing of lectins with mannose-substituted tetraphenylethenes based on aggregation-induced emission, Chem.-Asian J. 5 (2010) 817-824.

[36] H. Rosenfeld, J. Aniulyte, H. Helmholz, J. Liesiene, P. Thiesen, B. Niemeyer, A. Prange, Comparison of modified supports on the base of glycoprotein interaction studies and of adsorption investigations, J. Chromatogr. A 1092 (2005) 76-88.

[37] B.N. Murthy, N.H. Voelcker, N. Jayaraman, Evaluation of alpha-D-mannopyranoside glycolipid micelles-lectin interactions by surface plasmon resonance method, Glycobiology 16 (2006) 822-832.

[38] M.R. Eftink, C.A. Ghiron, R.A. Kautz, R.O. Fox, Fluorescence and conformational stability studies of staphylococcus nuclease and its mutants, including the less stable nuclease concanavalin-a hybrids, Biochem.-Us 30 (1991) 1193-1199.

[39] M.a. del Carmen Luzardo, M.a.E. Lanio, C. Alvarez, I.F. Pazos, S. Figueroa, V. Vérez, E.A.b. Disalvo, Aggregation induced by concanavalin A of lipid vesicles containing neoglycolipids, Colloids Surf. B 26 (2002) 281-289.

[40] T.K. Dam, R. Roy, S.K. Das, S. Oscarson, C.F. Brewer, Binding of multivalent carbohydrates to concanavalin a and dioclea grandiflora lectin: thermodynamic analysis of the multivalency effect, J. Biol. Chem. 275 (2000) $14223-14230$.

[41] M.C. Chervenak, E.J. Toone, Calorimetric analysis of the binding of lectins with overlapping carbohydrate-binding ligand specificities, Biochem.-Us 34 (1995) 5685-5695.

[42] C. Chen, H. Xu, Y.C. Oian, X.J. Huang, Glycosylation of polyphosphazenes by thiol-yne click chemistry for lectin recognition, RSC Adv. 5 (2015) $15909-15915$

[43] A.L. Zanocco, G. Gunther, F. Lemp, E.A. Lissi, Release of methylene blue from dioctadecyldimethylammonium chloride vesicles, J. Chem. Soc.-Perkin Trans. 2 (1998) 319-323.

[44] B.J. Frisken, C. Asman, P.J. Patty, Studies of vesicle extrusion, Langmuir 16 (2000) 928-933.

[45] K. Kinosita, S. Kawato, A. Ikegami, A theory of fluorescence polarization decay in membranes, Biophys. J. 20 (1977) 289-305.

[46] L.A. Chen, R.E. Dale, S. Roth, L. Brand, Nanosecond time-dependent fluorescence depolarization of diphenylhexatriene in dimyristoyllecithin vesicles and the determination of microviscosity*, J. Biol. Chem. 252 (1977).

[47] C.P.S. Altamirano, Caracterización fisicoquímica de sistemas microheterogéneos en presencia de derivados de manosa y sus aplicaciones, Departamento de Orgánica y Fisicoquímica, Universidad de Chile, 2017.

[48] S.A. Sanchez, M.A. Tricerri, G. Gunther, E. Gratton, Laurdan Generalized Polarization: from cuvette to microscope, in: A. Méndez-Vilas, J. Diaz (Eds.), Modern Research and Educational Topics in Microscopy, FORMATEX, 2007, pp. 1007-1014

[49] D. Hoekstra, N. Duzgunes, J. Wilschut, Agglutination and fusion of globoside GL-4 containing phospholipid vesicles mediated by lectins and calcium ion, Biochem.-Us 24 (1985) 565-572.

[50] A. Sekiguchi, H. Yamauchi, A. Manosroi, J. Manosroi, M. Abe, Molecular interactions between phospholipids and glycolipids in a lipid bilayer, Colloids Surf. B 4 (1995) 287-296.

[51] A. Engel, S.K. Chatterjee, A. Al-Arifi, P. Nuhn, Influence of spacer length on the agglutination of glycolipid-incorporated liposomes by ConA as model membrane, J. Pharm. Sci. 92 (2003) 2229-2235.

[52] E.M. Munoz, J. Correa, R. Riguera, E. Fernandez-Megia, Real-time evaluation of binding mechanisms in multivalent interactions: a surface plasmon resonance kinetic approach, J. Am. Chem. Soc. 135 (2013) 5966-5969. 
[53] T. Mori, M. Toyoda, T. Ohtsuka, Y. Okahata, Kinetic analyses for bindings of concanavalin A to dispersed and condensed mannose surfaces on a quartz crystal microbalance, Anal. Biochem. 395 (2009) 211-216.

[54] G.T. Noble, S.L. Flitsch, K.P. Liem, S.J. Webb, Assessing the cluster glycoside effect during the binding of concanavalin A to mannosylated artificial lipid rafts, Org. Biomol. Chem. 7 (2009) 5245-5254.

[55] J.J. Lundquist, E.J. Toone, The cluster glycoside effect, Chem. Rev. 102 (2002) 555-578.
[56] Y. Terada, H. Seto, Y. Hoshino, T. Murakami, S. Shinohara, K. Tamada, Y. Miura, SPR study for analysis of a water-soluble glycopolymer interface and molecular recognition properties, Polym. J. 49 (2017) 255-262.

[57] M. Reynolds, S. Pérez, Thermodynamics and chemical characterization of protein-carbohydrate interactions: the multivalency issue, C.R. Chim. 14 (2011) 74-95.

[58] R.J. Pieters, Maximising multivalency effects in protein-carbohydrate interactions, Org. Biomol. Chem. 7 (2009) 2013-2025. 